\title{
Correlations of Spectral Accretion Signatures in Young Binaries
}

\author{
Lisa Prato \\ UCLA, Dept. of Physics \& Astronomy, Math Sciences 8371, Los \\ Angeles, CA 90095-1562, USA \\ Jean-Louis Monin \\ Observatoire de Grenoble, Laboratoire d'Astrophysique, BP 53, F-38041 \\ Grenoble cedex, France
}

\begin{abstract}
We review correlations of spectral accretion diagnostics in young binary star systems. Hydrogen emission lines at visual $(\mathrm{H} \alpha)$ and near-infrared $(\mathrm{Br} \gamma)$ wavelengths indicate the presence of active accretion flows onto young stars. We examine the simultaneity of this process in both components of close binaries (separations 100-1500 AU) and find that active accretion is not a random process in these systems: the two stars are typically both active or both inactive. Only $19 \%$ of our sample consists of mixed pairs in which just one component displays evidence of accretion, usually the primary. In systems with two active stars, the equivalent width and in particular the line flux of the primary star is generally dominant, indicating stronger accretion activity. We discuss accretion and disk dissipation processes in close binaries and propose that a circumbinary envelope model accounts for the correlated evolution of circumstellar disks around young binaries.
\end{abstract}

\section{Introduction}

With the completion of several binary frequency surveys in the first half of the last decade, the predominance of multiples in the nearby star forming regions (SFRs) was well established (Ghez et al. 1993; Leinert et al. 1993; Simon et al. 1995). From this result there naturally arises a variety of questions regarding the circumstellar (CS) disks in these systems, since CS disks are the presumed sites of planet formation and stellar multiplicity could impact their formation and evolution. Do both stars in young binaries have CS disks? If not, which is lacking, the primary or secondary disk? Do both disks accrete and evolve at similar rates? The study of CS disks and accretion in pre-main-sequence (PMS) binary systems addresses these issues, providing insight into the evolution, coevality, disk dissipation timescales and formation models of young multiple stars.

To study accretion activity in young stars, we observed $\mathrm{H} \alpha$ and $\mathrm{Br} \gamma$ hydrogen emission lines, which are produced in accretion column flows (Shu et al. 1994; Hartmann et al. 1994). Although these lines are variable (e.g., Basri \& Bertout 1993), they are well correlated with other accretion diagnostics, such as optical veiling, forbidden [OI] line emission and near-infrared color excesses (Hartigan et al. 1990; Edwards et al. 1993, hereafter E93). $\mathrm{H} \alpha$ emission at $6563 \AA$ is also produced in energetic winds from young stellar objects (YSOs), 
however, such winds are correlated with active accretion. The $\operatorname{Br} \gamma 2.167 \mu \mathrm{m}$ line is a particularly robust diagnostic, and is useful for the study of embedded, red objects (Prato \& Simon 1997, hereafter PS97; Muzerolle et al. 1998).

The separation distribution of PMS binaries in Taurus peaks at $\sim 40 \mathrm{AU}$ (Simon et al. 1995), corresponding to an angular separation of $0.3^{\prime \prime}$. Thus, high spatial resolution is a requirement for observing spectral features in both components of close systems if we wish to study the stellar properties of the majority of these pairs. The technological advances in spectroscopy at large ground-based facilities in the last decade have enabled this research. Tip-tilt and adaptive optics systems, such as exist at the University of Hawaii $88^{\prime \prime}$ and the CFHT $3.6 \mathrm{~m}$ telescopes, were required for the observations of the individual stars in the closest binaries (separations $\sim 100 \mathrm{AU}$ ). The advent of sensitive array spectrometers in the near-infrared (IR) has also facilitated this work.

In $\S 2$ we describe the data samples on which we base our analysis, define the criteria for active accretion and present the results. We discuss correlations of accretion signatures in $\S 3$. Our interpretations of the results appear in $\S 4$. We list some observational predictions in $\S 5$.

\section{Data}

\subsection{Samples}

The observations which we analyze here are from several sources. In their 1994 paper, Hartigan et al. (hereafter H94) published a study of the characteristics of a sample of 39 PMS binary pairs with separations from 400-6000 AU. They obtained spatially resolved, i.e. for each component, $\mathrm{H} \alpha$ spectra for 28 systems in the Taurus and Orion SFRs. PS97 examined similar questions as those posed in this review $(\S 1)$ for a sample of 12 close binaries with separations of $40-360$ AU. Four of these, from the Taurus, Corona Australis and Ophiuchus SFRs, were resolved individually in the hydrogen $\mathrm{Br} \gamma$ line (separations of $180-360$ $\mathrm{AU})$. An additional unique $15 \mathrm{Br} \gamma$ systems, from the Lupus and Ophiuchus SFRs, appeared in Prato (1998) (separations 210-1000 AU). Data from a comparable sample of 15 systems in the Taurus SFR, separations 100-490 AU, are in preparation for publication (Prato et al. 2000). Brandner and Zinnecker (1997) studied accretion using the $\mathrm{H} \alpha$ diagnostic for a sample of 14 binaries (80-240 AU) in the southern SFRs Chameleon, Lupus and Ophiuchus. Monin et al. (1998; hereafter M98) and Duchêne et al. (1999; hereafter D99) performed similar studies on 15 systems in the Taurus SFR (130-830 AU).

\subsection{Criteria}

We distinguish actively accreting classical T Tauri stars (CTTs) from the weaklined T Tauris (WTTs) using the criteria described by Martín (1998) and Walter (1999). In place of the traditional $10 \AA \mathrm{H} \alpha$ cutoff, these authors propose that the emission line strength which defines a CTT is a function of spectral type. Thus, the limit for $\mathrm{K}$ stars is $5 \AA$, for M0-M2 type stars is $10 \AA$ and for later spectral type stars is $20 \AA$. For systems observed in the $\operatorname{Br} \gamma$ line, we adopt the criterion of $\sim 1 \AA$. 
To ensure a homogeneous sample of comparable binaries in our analysis, we eliminated, to the best of our knowledge, all higher order multiple systems with unresolved components. In order to avoid chance projections, we include only systems with separations $<11^{\prime \prime}$ (separations of the final sample were therefore $100-1500 \mathrm{AU}$ at the $\sim 140 \mathrm{pc}$ distance to the SFRs studied). In some cases systems were eliminated because they are not considered to be true binaries, such as GK Tau (H94; Prato et al. 2000). HBC 356/357 was discarded because it is probably not PMS (Martín 1998).

\subsection{Results}

In Figures 1 and 2 we present the $\mathrm{H} \alpha$ spectral line data in graphic form. The left panel of Figure 1 shows the secondary versus primary $\mathrm{H} \alpha$ equivalent widths from H94, M98 and D99 for objects primarily in the Taurus SFR. These data are converted to line fluxes and plotted in the right panel of Figure 1. There is a strong trend towards more active primary stars, particularly evident in the plots of line flux. The one object with the relatively large secondary line flux $\left(\sim 5 \times 10^{-16} \mathrm{Wm}^{-2}\right)$, DK Tau, is possibly a triple, a hypothesis supported by polarization observations presented by Jensen et al. (2000). RW Aur is included in this sample, although it does not appear in the right hand panel of Figure 1 or in Figure 2 since the primary and secondary line fluxes are relatively large, respectively, $120 \times 10^{-16}$ and $1.2 \times 10^{-16} \mathrm{Wm}^{-2}$. However, this system emphatically supports the trend towards dominant primaries. Figure 2 shows the secondary/primary line flux ratio as a function of the primary line flux. For systems with weak primary fluxes, significant scatter is present in the ratios. This may be understood as the result of division by a small number. The weaklined system J4872 has a ratio greater than unity which may only reflect that neither of the stars in this system is accreting. On the other hand, DK Tau again stands out in Figure 2 with a ratio $\sim 0.8$ and a large primary flux. Either this system is a triple, and the plotted ratio has to be refined by further higher angular resolution observations, or the secondary is significantly accreting in DK Tau, contrary to most of the other systems studied.

The $\operatorname{Br} \gamma$ data are plotted in Figures 3 and 4; the results are similar to those shown in Figures 1 and 2, i.e. the primary star equivalent widths and line fluxes tend to dominate. The right hand panel of Figure 4 is a blowup of part of the left hand panel. The only target with an appreciable secondary $\mathrm{Br} \gamma$ line flux is Haro 6-37, a triple which was intentionally included in this plot (although not in the analysis discussed below) for illustration. Four systems appear to have $\mathrm{Br} \gamma$ emission in the secondaries only; these are all mixed systems, discussed below (see Table 1).

The CTT and WTT nature of the binary components studied is summarized in Table 1. The Taurus sample includes a majority of pairs from the Taurus SFR and three systems from Orion. The Southern sample is composed of targets from Ophiuchus, Lupus, Corona Australis and Chameleon. We list the uncorrected and corrected percentages of mixed systems; one set of corrections were made in the classification of mixed pairs on the basis of the $\mathrm{H} \alpha$ or $\mathrm{Br} \gamma$ emission line for the CTT component being very close to the fiducial cutoffs described above. Such systems were reclassified as two WTTs, i.e. WW. Other corrections were made for two systems with optically thick $\mathrm{K}-\mathrm{L}$ colors associated with both 

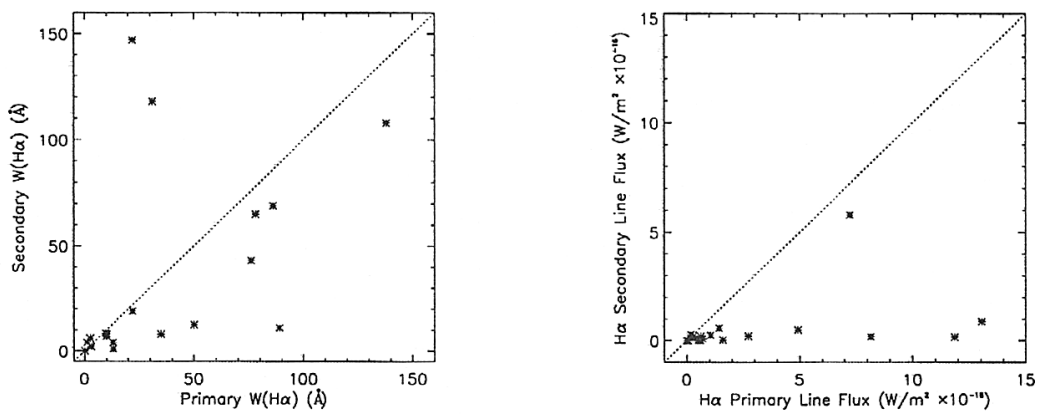

Figure 1. Left: Secondary versus primary $\mathrm{H} \alpha$ equivalent widths for the sample of Taurus objects from H94, M98 and D99. The dotted line indicates the location of objects for which the primary and secondary equivalent widths are equal.

Right: Secondary versus primary $\mathrm{H} \alpha$ line fluxes for the same sample of objects in the left panel. The dotted line indicates the location of objects for which the primary and secondary line fluxes are equal.

Table 1. Component Star Characterization

\begin{tabular}{cc}
\hline \hline Taurus Sample: & $\frac{\text { Southern Sample: }}{7 \mathrm{WW}}$ \\
$12 \mathrm{CC}$ & $10 \mathrm{CC}$ \\
$4 \mathrm{CW}(2 \mathrm{marginal})$ & $4 \mathrm{CW}(1 \mathrm{marginal})$ \\
$1 \mathrm{WC}$ & $6 \mathrm{WC}(2 \mathrm{CC}$ from colors $)$ \\
& $35 \%$ mixed uncorrected \\
$21 \%$ mixed uncorrected & $24 \%$ mixed corrected \\
\hline \hline Total sample (Taurus+Southern): 53 systems & $19 \%$ mixed corrected \\
\hline \hline $28 \%$ mixed uncorrected & \\
\hline \hline
\end{tabular}

components, although the binaries appear to have mixed spectral signatures. These were reclassified as two CTTs, i.e. CC (E93; PS97).

\section{Correlations}

The total number of systems in the sample we analyze here is 53, 24 from the Taurus SFR and 29 from the southern SFRs. In Table 1 we present the statistics for these regions separately. The southern regions have a higher fraction of mixed pairs. Given the small numbers in the samples, this may simply be a random fluctuation. However, it may also reflect physical differences in the properties of the different SFRs, such as stellar density and age. Much larger samples would be required to study this distinction at a high level of confidence.

The data presented in Table 1 show that only $28 \%$ of the systems in the uncorrected sample are mixed WC or CW pairs. This suggests that the component stars in PMS binaries in the separation range studied do not evolve 


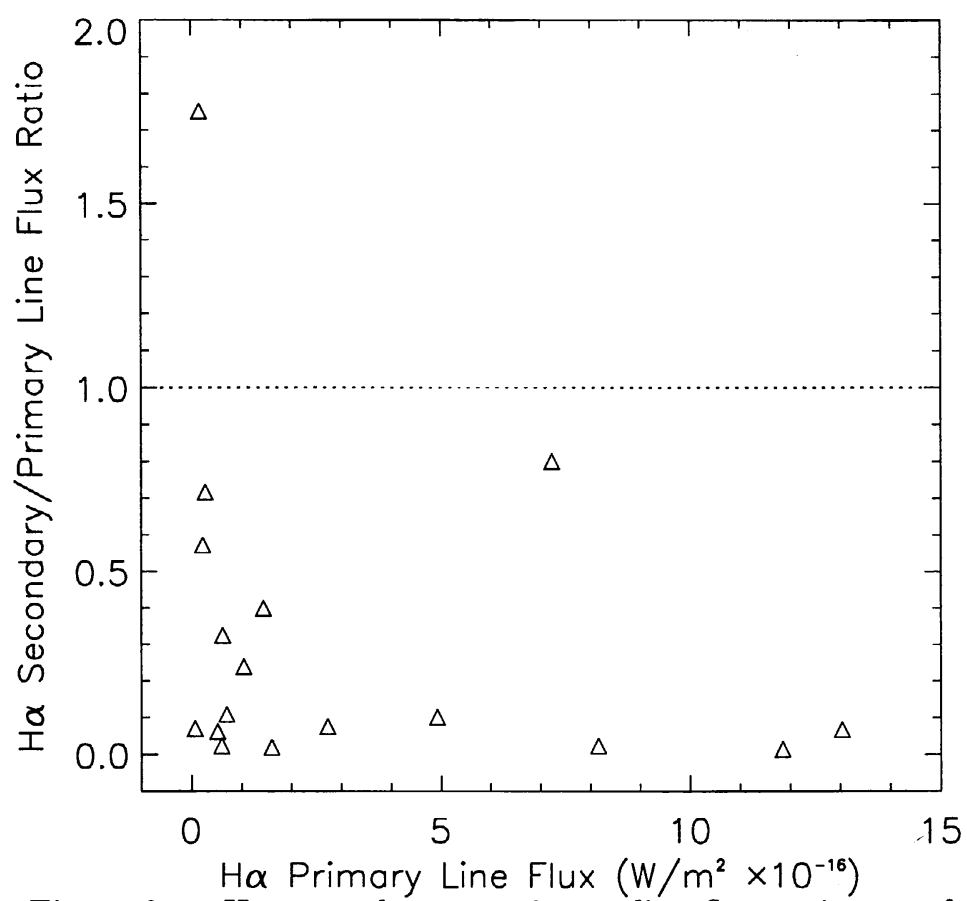

Figure 2. $\mathrm{H} \alpha$ secondary to primary line flux ratio as a function of the primary line flux. The scatter observed for small primary fluxes probably results from small number division, thus it is likely that neither component in the system J4872, with a ratio of $\sim 1.7$, is accreting. DK Tau appears out of place in the center of the plot; we speculate that this system may be a triple. The dotted line indicates the location of objects for which the primary and secondary line fluxes are equal.

independently of each other, consistent with the results of PS97 and D99. It appears that both stars in young, close binary systems accrete simultaneously. At some point, accretion ceases at nearly the same time in both components and the CS disks dissipate on relatively short time scales (see Simon \& Prato 1995). Modelling of random pairing between CTT and WTT stars has shown that stochastic processes do not account for the large number of pure WW and CC systems (PS97); rather, a shared physical environment seems to regulate the evolution of these binaries.

In the corrected sample, less than one fifth of the pairs are mixed, further strengthening our conclusions. The first corrections described in $\S 2.3$ shift pairs with one CTT member into the pure WW category, based on a borderline CTT equivalent width (i.e. within a few $\AA$ of the cutoff). The other correction described in $\S 2.3$, which shifts mixed pairs into the pure CC category on the basis of an optically thick near-IR color observed in the WTT component, introduces a qualitatively different diagnostic based on photometry. YSOs with weak-lined hydrogen emission and optically thick near-IR colors suggest passive CS disks which have ceased accreting and are evolving into WWs. Since line emission 


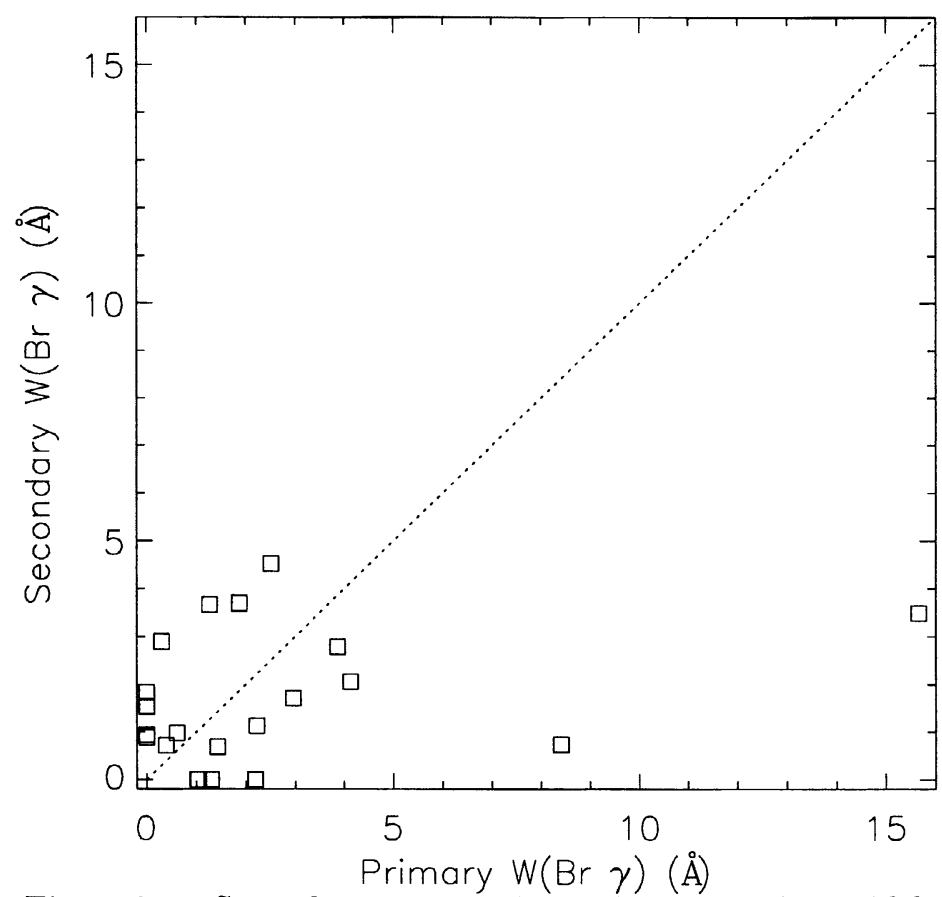

Figure 3. Secondary versus primary Br $\gamma$ equivalent widths for a sample of Taurus and southern SFR systems. The dotted line indicates the location of objects for which the primary and secondary equivalent widths are equal.

attributable to accretion onto the central star is a highly variable process, it could also be that WTT components with optically thick passive disks are in a quiescent accretion state. However, in mixed systems, the CTT component typically displays relatively weak emission in comparison to the components in pure CCs, supporting the scenario in which mixed pairs are likely to be in transition to WWs, rather than CC systems with one star in a dormant state.

Figure 5, adapted from PS97, shows the near-IR K-L versus mid-IR K-N color plane. Both single and (spatially unresolved) binary systems are plotted, as circles and triangles, respectively; the symbol size is proportional to the $\mathrm{H} \alpha$ equivalent width, $\mathrm{W}(\mathrm{H} \alpha)$. WTT singles and doubles have optically thin colors and occupy the lower left-hand corner of the diagram, as expected. However, a number of WW binary systems, as well as some single WTTs, are located in the upper right-hand area of the plot, characteristic of stars with near and mid-IR optically thick colors, implying the presence of a disk. The data plotted, for objects in the Taurus SFR, are from H94, Simon \& Prato (1995), Kenyon \& Hartmann (1995) and Herbig \& Bell (1988); the binaries included have a separation range of $\sim 40-400$ AU. Kenyon et al. (1996) and Armitage et al. (1999) have modelled the evolution of stars in $\mathrm{K}-\mathrm{L} / \mathrm{K}-\mathrm{N}$ space. In the models of Kenyon et al., as the CS disks begin to dissipate, outwards from an inner gap, the objects appear redder. Following the Kenyon et al. evolutionary tracks in 

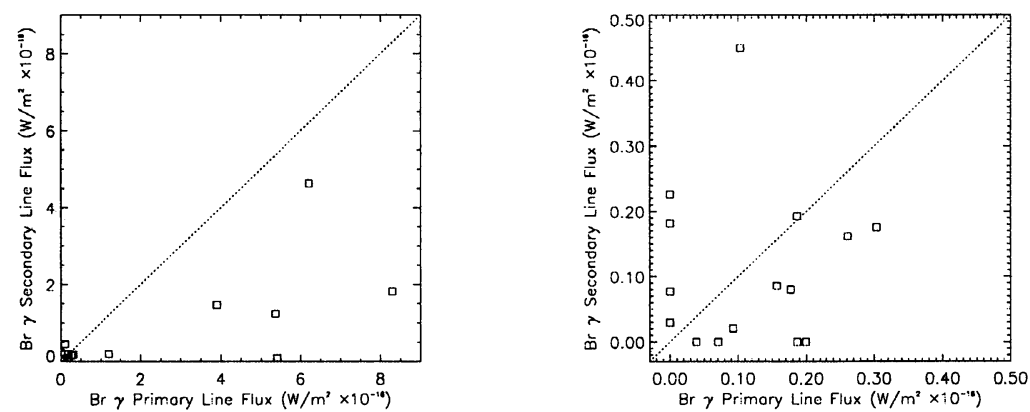

Figure 4. Left: Secondary versus primary $\mathrm{Br} \gamma$ line fluxes for the same sample of objects in Figure 3. The dotted line indicates the location of objects for which the primary and secondary line fluxes are equal. Right: A blowup of the lower left-hand corner of the left panel. Haro $6-37$, the object with the largest secondary line flux, is a triple. We include it here to show how a triple system contrasts with the simple doubles.

Figure 5, the system first tends to move diagonally towards the upper right of the diagram, then down to the lower right-hand sectors, then horizontally left to the WTT region. Thus, in this model, we expect to see some very red systems with little or no hydrogen emission, as observed, because the warm material in the inner disk has dissipated.

\section{Interpretation}

We propose that a tenuous, optically thin circumbinary (CB) envelope surrounds both objects in a close CC binary, analogous to CS envelopes around single stars (Calvet et al. 1994). Such an envelope could feed and simultaneously maintain the disks of both stars. Accretion from the disks onto the central stars gives rise to the emission lines associated with both stars. Since the average angular momentum of an envelope is low, material falls at least equally onto both disks or, more probably, preferentially onto the disk of the primary (Bate 2000), in which case we expect case the primary star's CS disk to be more massive than the secondary's. Consequently, if the rate of accretion from a disk onto a central star is proportional to the disk mass, then the primary stars should experience larger accretion rates, as observed. Once the envelope material has been depleted, the remaining accretion lifetime of both the circumprimary and circumsecondary disks would be similar, since $\tau_{d i s k}=\mathrm{M}_{d i s k} / \dot{\mathrm{M}}$, where $\dot{\mathrm{M}}$ is the accretion rate of the disk onto the central star (D99). Simon \& Prato (1995) show that the disk dissipation times may be as short as $\lesssim 10^{5}$ years, indicating that the disks do not survive long once the reservoir of supply material, the envelope, is gone. We base our interpretation on systems with separations of 100-1500 AU. The fraction of mixed systems is higher in wider pairs (H94; PS97), thus, it is unlikely that this envelope model holds for wide binaries, as discussed in PS97.

A CB (or CS) envelope model accounts for the fact that a comparison of measured disk masses and observed accretion rates indicates that most observed 


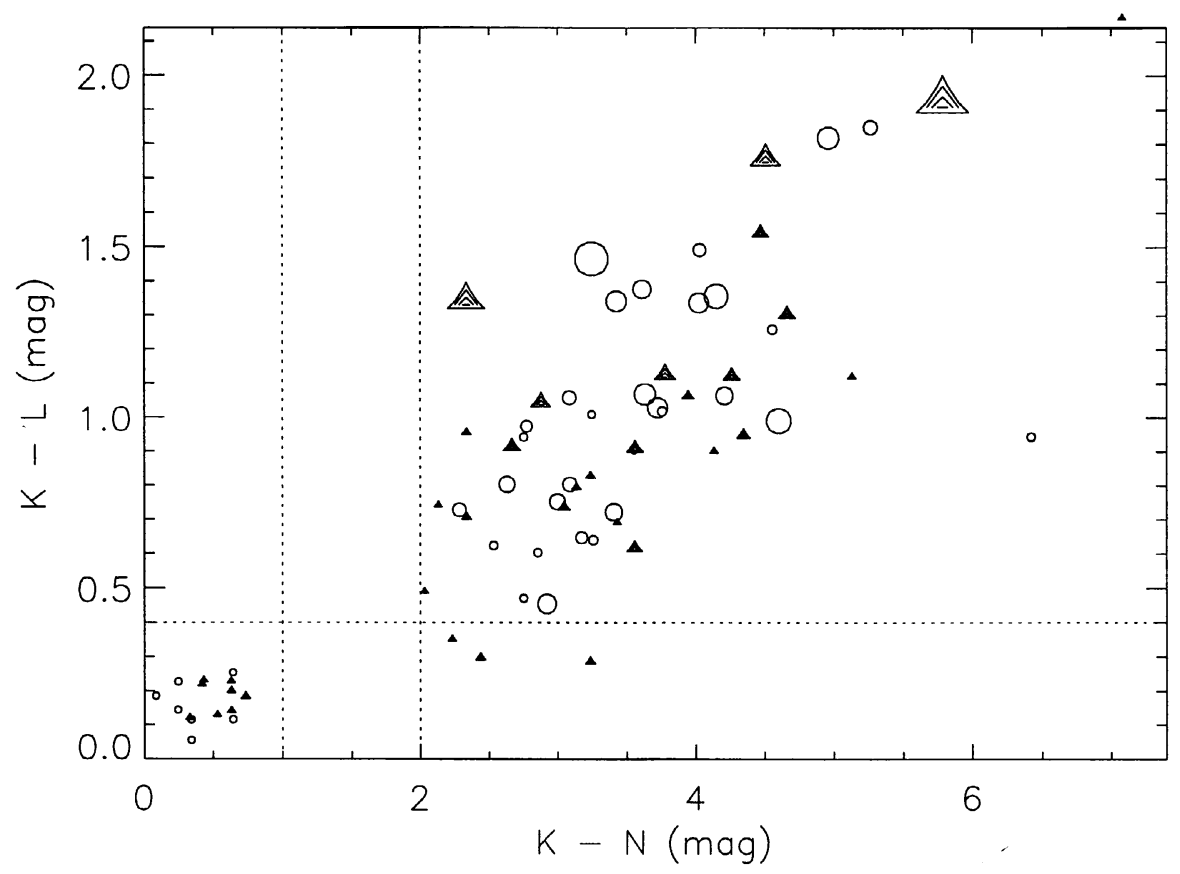

Figure 5. The $\mathrm{K}-\mathrm{L}$ versus $\mathrm{K}-\mathrm{N}$ plane. Circles are single stars and triangles are (unresolved) binaries. The dotted lines delineate the optically thick / optically thin cutoffs for the two colors, 0.4 mag for $\mathrm{K}-\mathrm{L}$ and 1-2 for $\mathrm{K}-\mathrm{N}$. The symbol size is proportional to the $\mathrm{W}(\mathrm{H} \alpha)$.

disks ought to have accreted and dissipated long ago, given the stellar ages (e.g. Strom et al. 1989; PS97). Thus, a mechanism that accounts for disk feeding is critical. The presence of a disk is not a strong function of age in PMS systems (e.g., Simon \& Prato 1995). We propose that the disk lifetimes depend on the amount of material originally contained in the envelope. Millimeter observations have not detected these envelopes in large numbers, athough there is some indication that complex CS and CB environments exist and may harbor structures which perform an equivalent function (see Stapelfeldt \& Ménard 2000). Based on the parameters given in Calvet et al. (1994), a rough estimate suggests that sufficient material to feed the disks may be present in an envelope, but may lie below the sensitivity limits of most millimeter observations to date. Furthermore, a number of CS envelopes have been detected, indicating that they are at least more common than rare structures such as CB disks. Bate (2000) argues that a CB disk has high angular momentum, and would preferentially accrete onto the secondary stars, contrary to the trend of the observations discussed here. 


\section{Observational Predictions}

We conclude by listing several predictions, consistent with the model we have discussed, which may be tested observationally.

$\Rightarrow$ If $\mathrm{M}_{\text {disk }} \propto \dot{\mathrm{M}}$ explains the similar accretion timescales in primaries and secondaries, then this should hold for single stars as well as for binaries. This correlation is currently difficult to explore because of the uncertainties in the young star parameters required to derive the accretion rates. Improved mass estimates for $\mathrm{T}$ Tauri stars based on dynamical observations and the next generation of theoretical models should help to establish or disprove such a relation.

$\Rightarrow$ The few systems with very strong accretion signatures in the secondaries, yet none or only weak signatures in the primaries, could be surveyed for CB rings, as predicted by Bate (2000). Alternatively, these may not be physically related binaries.

$\Rightarrow$ If mixed systems are in the process of dissipation and have only just stopped or are stopping final accretion, then both stars in such binaries should have near and mid-IR optically thick colors indicative of passive CS disks. The same should be true for all CC systems.

\section{References}

Armitage, P. J., Clarke, C. J., \& Tout, C. A. 1999, MNRAS, 304, 425

Basri, G., \& Bertout, C. 1993, Protostars and Planets III, 543

Bate, M. 2000, in press

Brandner, W., \& Zinnecker, H. 1997, A\&A, 321, 220

Calvet, N., Hartmann, L., Kenyon, S. J., \& Whitney, B. A. 1994, ApJ, 434, 330

Duchêne, G., Monin, J.-L., Bouvier, J., \& Ménard, F. 1999, A\&A, 351, 954 (D99)

Ghez, A. M., Neugebauer, G., \& Matthews, K. 1993, AJ, 106, 2005

Edwards, S., Ray, T., \& Mundt, R. 1993, Protostars and Planets III, 567 (E93)

Hartigan, P., Hartmann, L., Kenyon, S. J., Strom, S. E., \& Skrutskie, M. F. 1990, ApJ, 354, L25

Hartigan, P., Strom, K. M., \& Strom, S. E. 1994, ApJ, 427, 961 (H94)

Hartmann, L., Hewett, R., \& Calvet, N. 1994, ApJ, 426, 669

Herbig, G. H., \& Bell, K. R. 1988, Lick Observatory Bulletin No. 1111

Jensen, E. L. N., Donar, A. X., \& Mathieu, R. D. 2000, in Birth and Evolution of Binary Stars, poster proceedings of IAU Symp. No. 200, ed. B. Reipurth \& H. Zinnecker, 85

Kenyon, S. J., \& Hartmann, L. 1995, ApJS, 101, 117

Kenyon, S. J., Yi, I., \& Hartmann, L. 1996, ApJ, 462, 439

Leinert, Ch., Zinnecker, H., Weitzel, N., Christou, J., Ridgway, S. T., Jameson, R., Haas, M., \& Lenzen, R. 1993, A\&A, 278, 129

Martín, E. L. 1998, AJ, 115, 351

Monin, J.-L., Menard, F., \& Duchene, G. 1998, A\&A, 339, 113 (M98)

Muzerolle, J., Hartmann, L., \& Calvet, N. 1998, AJ, 116, 2965 
Prato, L., \& Simon, M. 1997, ApJ, 474, 455 (PS97)

Prato, L. A. 1998, Ph.D. Thesis, SUNY Stony Brook

Prato, L., Greene, T. G., Simon, M., \& Beck, T. L. 2000, in preparation

Shu, F., Najita, J., Ostriker, E., Wilkin, F., Ruden, S., \& Lizano, S. 1994, ApJ, 429,781

Simon, M., \& Prato, L. 1995, ApJ, 450, 824

Simon, M., Ghez, A. M., Leinert, Ch., Cassar, L., Chen, W. P., Howell, R. R., Jameson, R. F., Matthews, K., Neugebauer, G., \& Richichi, A. 1995, ApJ, 443, 625

Stapelfeldt, K., \& Ménard, F. 2000, this volume

Strom, K. M., Strom, S. E., Edwards, S., Cabrit, S., \& Skrutskie, M. F. 1989, AJ, 97, 1451

Walter, F. M. 1999, in Solar and Stellar Activity: Similarities and Differences, ASP Conference Series 158, ed. C. J. Butler \& J. G. Doyle, 87

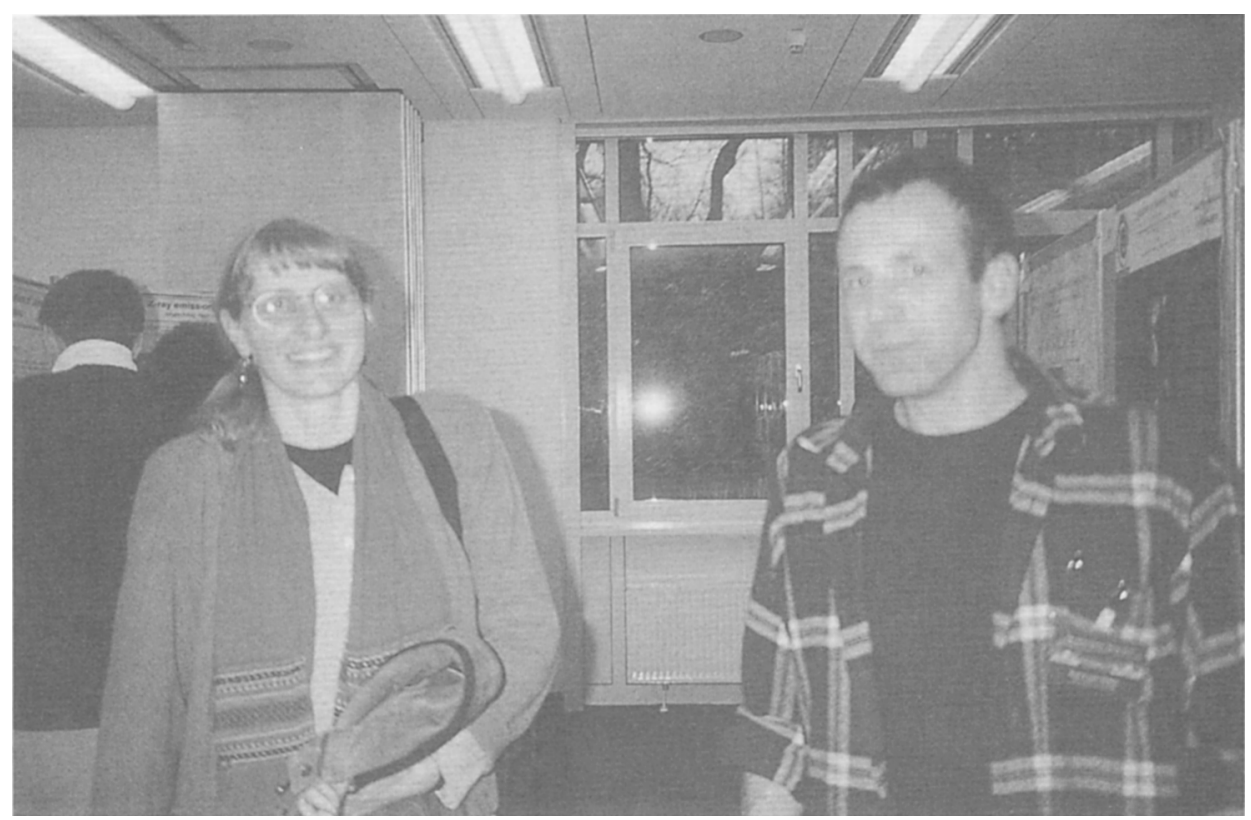

Lisa Prato and Jean-Louis Monin 